\title{
Multicenter Analysis of mFOLFOX6 with Oxaliplatin Stop-and-Go Strategy Using Oral Uracil-Tegafur with Leucovorin for Unresectable Colorectal Cancer in Elderly Patients
}

\author{
Tetsu Yamamoto $^{1 *}$, Kuniyuki Katano ${ }^{2,3}$, Shinichi Sugimoto ${ }^{4}$, Akiyoshi Kanazawa ${ }^{5}$, Eiji Hira ${ }^{1}$, \\ Hiroshi Takeda ${ }^{4}$, Yoshitoshi Sato ${ }^{6}$, Yutaka Yamashiro ${ }^{7}$, Yoshitsugu Tajima ${ }^{1}$, Masahide Ikeguchi $^{3}$ \\ ${ }^{1}$ Department of Digestive and General Surgery, Faculty of Medicine, Shimane University, Izumo, Japan; ${ }^{2}$ Department of Surgery, \\ Saihaku Hospital, Saihaku, Japan; ${ }^{3}$ Division of Surgical Oncology, Faculty of Medicine, Tottori University, Yonago, Japan; ${ }^{4}$ De- \\ partment of Surgery, Shimane Prefectural Central Hospital, Izumo, Japan; ${ }^{5}$ Department of Gastroenterological Surgery, Osaka Red \\ Cross Hospital, Osaka, Japan; ${ }^{6}$ Department of Gastroenterological Surgery, Matsue Red Cross Hospital, Matsue, Japan; ${ }^{7}$ Department \\ of Gastroenterological Surgery, Tottori Red Cross Hospital, Tottori, Japan. \\ Email: ${ }^{*}$ 2t2@med.shimane-u.ac.jp
}

Received December 31 ${ }^{\text {st }}$, 2013; revised January $18^{\text {th }}$, 2014; accepted January $25^{\text {th }}, 2014$

Copyright (c) 2014 Tetsu Yamamoto et al. This is an open access article distributed under the Creative Commons Attribution License, which permits unrestricted use, distribution, and reproduction in any medium, provided the original work is properly cited. In accordance of the Creative Commons Attribution License all Copyrights (C) 2014 are reserved for SCIRP and the owner of the intellectual property Tetsu Yamamoto et al. All Copyright (C) 2014 are guarded by law and by SCIRP as a guardian.

\section{ABSTRACT}

Background: This study evaluated the tolerability and efficacy of intermittent oxaliplatin treatment based on mFOLFOX6 using oral uracil-tegafur(UFT) and leucovorin(LV) maintenance therapy in the treatment of elderly patients with advanced colorectal cancer. Methods: Ten non-elderly patients ( $<70$ years) and 8 elderly patients ( $>70$ years) with advanced/recurrent colorectal cancer were enrolled in this prospective, multicenter cooperative group clinical trial. The mFOLFOX6 regimen was administered for eight cycles with maintenance therapy with oral UFT/LV treatment until progression. In cases with disease progression, mFOLFOX6 was reintroduced. Results: Grade 2 peripheral neuropathy was noted in $30.0 \%$ and $25.0 \%$ of the elderly and non-elderly patients, respectively. The observed time to treatment failure (TTF) was 6.3 months in the elderly patients and 6.4 months in the non-elderly patients. The disease control rate was $83.3 \%$ in each group. Conclusion: Our new stop-and-go strategy using oral UFT/LV is well-tolerated and effective even in elderly patients.

\section{KEYWORDS}

\section{Colorectal Cancer; Oxaliplatin; Uracil-Tegafur (UFT); Leucovorin; Elderly Patient; Stop-and-Go}

\section{Introduction}

Colorectal cancer is one of the most common malignancies and the third leading cause of cancer death, with an increasing incidence in Japan [1]. FOLFOX therapy with oxaliplatin and infusional 5-Fluorouracil (5-FU)/levofolinate (LV) has extended the median overall survival of the patients with advanced colorectal cancer to 2 years and beyond, and this therapy is now widely accepted as a first-line treatment for unresectable/recurrent colorectal cancer [2].

*Corresponding author.
The incidence of colorectal cancer is increasing, especially in the elderly, in Japan. In the treatment of elderly patients with colorectal cancer, a decline of organ function associated with aging raises a great disadvantage because multi-drug chemotherapies cause severe adverse events. Although mFOLFOX6 therapy is well-tolerated and effective in elderly patients over 70 years old, discontinuation of treatment is sometimes necessary due to peripheral neuropathy [3].

Because oxaliplatin can lead to the development of specific cold-related dysesthesia and cumulative peripheral sensory neuropathy, many patients have to withdraw 
from the oxaliplatin-containing regimen. In addition, this neuropathy persists for a long time after discontinuance of the treatment. The reduction of oxaliplatin-related peripheral neuropathy is therefore important and some studies have reported strategies to prevent peripheral neuropathy which has been reported to be associated with oxaliplatin.

The OPTIMOX1 (Optimized Leucovorin [LV]-Fluorouracil [FU]-Oxaliplatin) and CONcePT (Combined Oxaliplatin Neurotoxicity Prevention Trial) studies demonstrated that a stop-and-go strategy can reduce the development of sensory neuropathy and also has an equivalent efficacy in comparison to the continuance of oxaliplatin-containing regimens until progression or unacceptable toxicity [4].

5 -FU is administered by bolus injection or by a continuous infusion in mFOLFOX6 and simplified LV5FU2 regimens. Continuous infusion requires a specialized pump, which is inconvenient for patients [5]. Furthermore, longterm venous access increases the risks of infection and venous thrombosis.

The oral fluoropyrimidine, UFT (tegafur-uracil), is an effective, well-tolerated and convenient alternative to i.v. 5 -FU and is widely used in the treatment of colorectal cancer [6]. Oral UFT/LV therapy may be an alternative to continuous or bolus injection of 5-FU and leucovorin therapy.

The San-in Study Group on colorectal cancer (SSCC) started multicenter studies on the treatment of unresectable advanced/recurrent colorectal cancer in 2006. The present study (SSCC-0701) was a prospective study and carried out to evaluate tolerability and efficacy of intermittent oxaliplatin treatment based on mFOLFOX6 using oral UFT/LV maintenance therapy in the treatment of elderly patients with advanced colorectal cancer.

\section{Methods}

\subsection{Patient Selection}

Patients who were confirmed to be eligible for this study fulfilled the eligibility criteria and received mFOLFOX6 therapy for unresectable advanced/recurrent colorectal at any of the three participating institutions of SSCC (Division of Surgical Oncology, Tottori University Faculty of Medicine; Department of Digestive and General Surgery, Shimane University Faculty of Medicine; and Department of Surgery, Shimane Prefectural Central Hospital) during the period from August 2007 to September 2008 were enrolled. All Patients were diagnosed as unresectable advanced/recurrent colorectal cancer by colonoscopy and CT scan.

The protocol was approved by the institutional ethics committees and this study was carried out according to the principles of the Declaration of Helsinki and Ethical Guidelines for Clinical Studies [7,8].
The eligibility criteria were as follows: histologically proven unresectable colorectal adenocarcinoma; $>1 \mathrm{~cm}$ or a non-measurable assessable lesion; with an adequate function of the bone marrow, liver, and kidney; Eastern Cooperative Oncology Group (ECOG) performance status (PS) <2; age > 20 years old at the time of enrollment; expected survival time $>12$ months; and no previous chemotherapy for unresectable colorectal cancer. Previous adjuvant chemotherapy was required to have been completed at least 6 months before inclusion Patients with multiple malignancies, prior radiotherapy, pregnancy or lactation, symptomatic peripheral neuropathy, history of serious drug hypersensitivity, or any comorbidities that could influence the outcome were excluded from the analysis. Written informed consent was obtained from all participating patients.

\subsection{Treatment Schedule}

A schematic illustration of the novel stop-and-go regimen is displayed in Figure 1(a). The outline of mFOLFOX6 administration, in which the dose of oxaliplatin was reduced from $100 \mathrm{mg} / \mathrm{m}^{2}$ to $85 \mathrm{mg} / \mathrm{m}^{2}$, is shown in Figure 1(b). Patients received mFOLFOX6 for eight cycles (a 2-hour intravenous infusion of $85 \mathrm{mg} / \mathrm{m}^{2}$ oxaliplatin plus $200 \mathrm{mg} / \mathrm{m}^{2}$ leucovorin followed by a 400 $\mathrm{mg} / \mathrm{m}^{2} 5$-FU bolus and 46-hour infusion of $2400 \mathrm{mg} / \mathrm{m}^{2}$ every 2 weeks) and then received oral daily UFT/LV treatment, consisting of UFT $300 \mathrm{mg} / \mathrm{m}^{2} /$ day and LV 75 $\mathrm{mg}$ /day orally in three divided doses taken at approximately 8-hour intervals for 28 days every 35 days, until progression (Figure 1(c)). When tumor progression was evident, mFOLFOX6 therapy was reintroduced (Figure 1(a)). A $5-\mathrm{HT}_{3}$ antagonist and a steroid were administered as premedication. An oral steroid was given for 3 days from day 2 after the start of chemotherapy. The duration of one cycle was 2 weeks. The use of implantable ports and disposable pumps allowed chemotherapy to be administered on an outpatient basis.

\subsection{Evaluation Criteria}

Each treatment cycle was only started after confirming all of the following criteria.

1) Hematological toxicity: leukocyte count $>3000 /$ $\mathrm{mm}^{3}$ and platelet count $>75,000 / \mathrm{mm}^{3}$.

2) Non-hematological toxicity: Grade 2 or less according to the Common Terminology Criteria for Adverse Events (CTCAE) v3.0, and Grade 1 or less for peripheral neuropathy [9].

3) The drug administration could be postponed if any of the patient's condition deviate from the criteria or at the investigator's discretion following harmful events, such as a rapid decrease in the leukocyte or platelet count 
Stop and go regimen

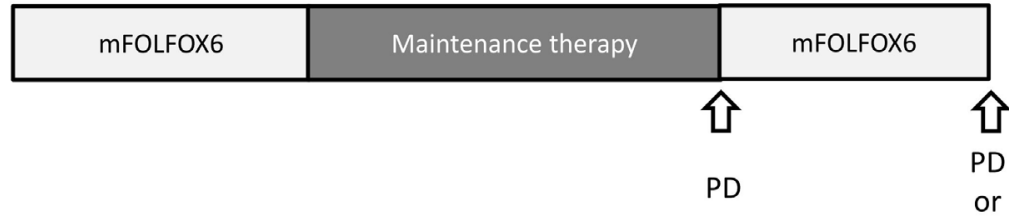

Discontinuation criteria

(a)

Modified FOLFOX6 (induction and reintroduction)

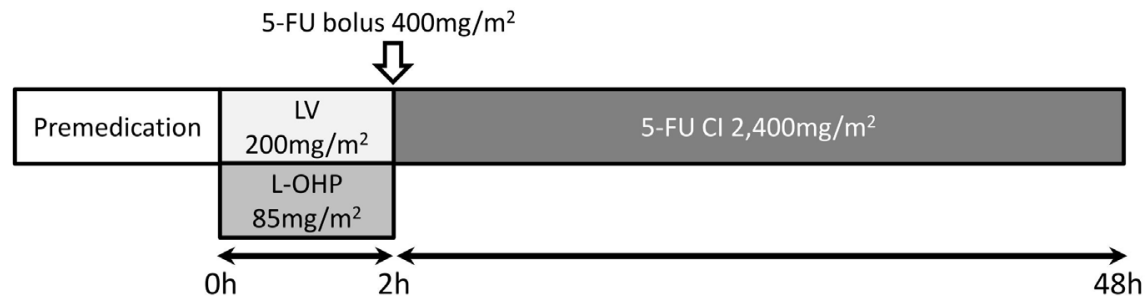

(b)

UFT / leucovorin (maintenance therapy)

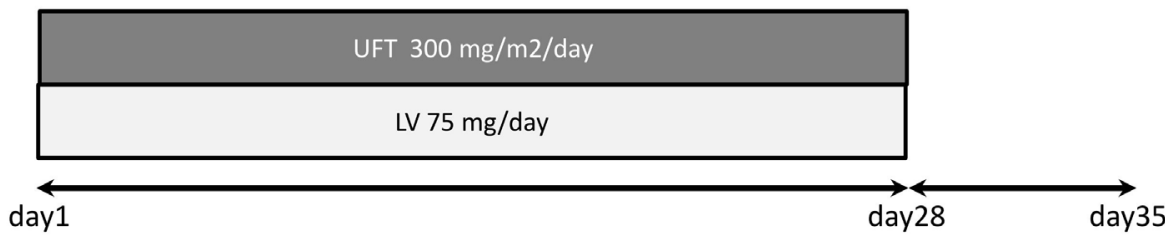

(c)

Figure 1. Chemotherapy regimens: (a) Stop and go regimen; (b) modified FOLFOX6 (induction and reintroduction); and (c) UFT/LV (maintenance therapy); UFT, uracil-tegafur; LV, leucovorin.

and the occurrence of jaundice, irrespective of any other criteria deviation. The subsequent course could be postponed for up to 21 days (excluding the scheduled day of starting administra-tion) and the study was discontinued when the postponement of treatment exceeded this period.

Computed tomography scans of measurable lesions were assessed within 28 days prior to the beginning of the treatment, repeated every four cycles or 2 months.

\subsection{Dose Reduction}

A dose reduction method was introduced to continue mFOLFOX6 therapy in elderly patients when grade 3 neutropenia or thrombocytopenia occurred, while oxaliplatin was not reduced [3], that is, only bolus administration of 5-FU was stopped without any reduction of the other drugs.

\subsection{Endpoints}

The primary assessment criterion was time to treatment failure (TTF). The second assessments were antitumor effect (response rate, tumor stabilization rate, and dura- tion of response), safety, and efficacy in elderly patients. Adverse events and therapeutic efficacy were assessed according to the CTCAE v3.0 and the RECIST guidelines (version 3) [10]. Extramural review was performed for judgment of the eligibility and handling of registered patients, as well as for safety and efficacy assessment.

\subsection{Statistical Analysis}

The Chi-square test, Fisher's exact probability test, and the Mann-Whitney U test were used to compare patient characteristics, treatment status, adverse events, and antitumor effects. A probability (P) value of less than 0.05 was considered to be statistically significant for comparisons between the elderly and non-elderly groups. The Kaplan-Meier method was used to estimate TTF. Statistical analyses were performed using the JMP ver. 5.0.1 software package (SAS institute Inc, Cary, NC).

\section{Results}

\subsection{Patient Characteristics}

A total of 18 patients were enrolled in this study, includ- 
ing 10 non-elderly patients and 8 elderly patients. Their median age was 66.5 years old (range: 48 - 82); 61.0 years old (range 48 - 68) in the non-elderly group and 77.0 years old (range 71 - 82) in the elderly group. The baseline characteristics of the 18 eligible and treated patients are summarized in Table 1. Six patients (60\%) in the non-elderly group and 4 patients (50\%) in the elderly group were male. Performance status was less than 2 in all patients. Six patients (60\%) and 7 patients (88\%) in the non-elderly and elderly groups had colon cancer as primary tumor, respectively. Twelve patients had tumors in the liver, 4 patients in the lung, and 3 patients in the lymph nodes.

\subsection{Treatment Status}

The total number of mFOLFOX6-treatment cycles administered was 188, including 129 cycles of oral UFT/LV as maintenance therapy. The median number of mFOLFOX6-treatment cycles was 9.0 (range 1 - 27) in the non-elderly group and 8.0 cycles (range 1 - 17) in the elderly group. Nine patients (50\%) received the maintenance treatment. The median number of cycles of maintenance therapy was 11 cycles (range: 9 - 27) in the non-elderly group and 17 cycles (range: 11 - 17) in the elderly group. Only 3 patients (16.7\%), 2 patients in the non-elderly group and one in the elderly group, required oxaliplatin reintroduction due to disease progression.

Three patients were introduced an alternative regimen with bevacizumab, where one patient had residual neurotoxicity and 2 patients withdrew their consent.

\subsection{Adverse Events}

Grade 2 anemia occurred in $50.0 \%$ of the elderly patients, and the incidence was higher than that in the non-elderly patients $(0 \%)(\mathrm{P}=0.015)$. The frequency and grade of the other adverse events were similar in the two groups (Table 2). Although grade 3 neutropenia occurred in 5 patients (4 non-elderly patients and one elderly patient), treatment could be continued without reducing the dose of oxaliplatin by deleting the bolus infusion of 5-FU. Grade 2 peripheral neuropathy occurred in $30.0 \%$ and $25.0 \%$ of the non-elderly and elderly patients, respecttively. Relation between the incidence of neuropathy and the number of treatment cycles is shown in Table 3. Although grade 2 neuropathy occurred in 4 patients in the non-elderly group and 1 patient in the elderly group during the $8^{\text {th }}$ cycles of mFOLFOX6, the occurrence of neuropathy was decreased after the $9^{\text {th }}$ cycles and grade 3 neuropathy was not recognized in this study.

\subsection{Duration of Treatment}

The median TTF was 6.4 months in the non-elderly group and 6.3 months in the elderly group (Figure 2). No significant difference was found in TTF between the 2 groups. Nine patients could not continue the initial mFOLFOX6 treatment. The reasons for the discontinuation of the mFOLFOX6 treatment were tumor progression, allergy and the patient's choice.

Six patients in the non-elderly patients group and $3 \mathrm{pa}-$ tients in the elderly group received the maintenance therapy. The median duration of maintenance therapy with oral UFT/LV was 9 cycles in the non-elderly group and 17 cycles in the elderly group. The reason for discontinuation of maintenance therapy was the progressive disease in all patients. No severe adverse effect was recognized during maintenance therapy.

Table 1. Baseline characteristics of the two study groups.

\begin{tabular}{|c|c|c|c|c|}
\hline & $\begin{array}{c}<70 \text { Years } \\
(\mathrm{n}=10)\end{array}$ & & $\begin{array}{c}\geqq 70 \text { Years } \\
\quad(\mathrm{n}=8)\end{array}$ & \\
\hline Age, y (median) & $61(48-68)$ & & $77(71-82)$ & \\
\hline Male & 6 & $(60 \%)$ & 4 & $(50 \%)$ \\
\hline Female & 4 & $(40 \%)$ & 4 & $(50 \%)$ \\
\hline \multicolumn{5}{|l|}{ PS (ECOG), n } \\
\hline 0 & 6 & $(60 \%)$ & 4 & $(50 \%)$ \\
\hline 1 & 4 & $(40 \%)$ & 4 & $(50 \%)$ \\
\hline 2 & 0 & $(0 \%)$ & 0 & $(0 \%)$ \\
\hline \multicolumn{5}{|l|}{ Primary tumor, $\mathrm{n}$} \\
\hline Colon & 6 & $(60 \%)$ & 7 & $(88 \%)$ \\
\hline Rectum & 4 & $(40 \%)$ & 1 & $(13 \%)$ \\
\hline Both & 0 & $(0 \%)$ & 0 & $(0 \%)$ \\
\hline \multicolumn{5}{|l|}{ Target lesions, $\mathrm{n}$} \\
\hline Liver & 6 & $(60 \%)$ & 6 & $(75 \%)$ \\
\hline Lung & 3 & $(30 \%)$ & 1 & $(13 \%)$ \\
\hline Lymph node & 1 & $(10 \%)$ & 2 & $(25 \%)$ \\
\hline Previous surgery, n & 7 & $(70 \%)$ & 6 & $(75 \%)$ \\
\hline $\begin{array}{c}\text { Prior adjvant } \\
\text { chemotherapy, } \mathrm{n}\end{array}$ & 0 & $(0 \%)$ & 4 & $(50 \%)$ \\
\hline
\end{tabular}

Abbreviations: PS, performance status; ECOG, Eastern Cooperative Oncology Group. Values in parentheses are percentages of row totals.

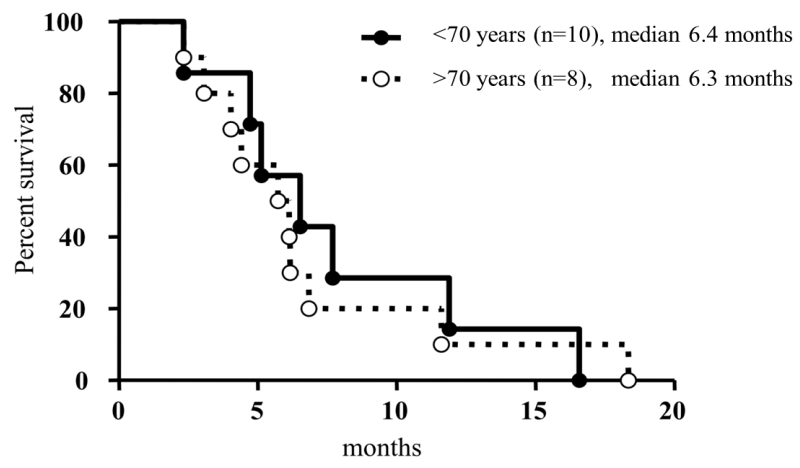

Figure 2. Kaplan-Meier curves of time to treatment failure (TTF). The median TTF was 6.4 months and 6.3months in non-elderly patients group $(O)$ and elderly patients group (O), respectively. 
Table 2. Major adverse events of mfolfox6 with oxaliplatin stop-and-go strategy.

\begin{tabular}{|c|c|c|c|c|c|c|c|c|c|}
\hline & \multicolumn{4}{|c|}{$<70$ Years $(\mathrm{n}=10)$} & \multicolumn{4}{|c|}{$\geqq 70$ Years $(n=8)$} & \multirow[t]{2}{*}{ P Values* } \\
\hline & \multicolumn{2}{|c|}{ Grade 2} & \multicolumn{2}{|c|}{ Grade 3} & \multicolumn{2}{|c|}{ Grade 2} & \multicolumn{2}{|c|}{ Grade 3} & \\
\hline & $\mathrm{n}$ & & $\mathrm{n}$ & & $\mathrm{n}$ & & $\mathrm{n}$ & & \\
\hline Leukocytopenia & 3 & $(30.0 \%)$ & 4 & $(40.0 \%)$ & 3 & (26.7\%) & 1 & $(12.5 \%)$ & 0.40 \\
\hline Neutropenia & 0 & $(0.0 \%)$ & 4 & $(40.0 \%)$ & 0 & $(0.0 \%)$ & 3 & (37.5\%) & 0.38 \\
\hline Anemia & 0 & $(0.0 \%)$ & 0 & $(0.0 \%)$ & 4 & $(50.0 \%)$ & 0 & $(0.0 \%)$ & 0.02 \\
\hline Thrombocytopenia & 3 & (30.0\%) & 0 & $(0.0 \%)$ & 2 & $(25.0 \%)$ & 0 & $(0.0 \%)$ & 0.91 \\
\hline Nausea & 1 & $(10.0 \%)$ & 0 & $(0.0 \%)$ & 2 & $(25.0 \%)$ & 0 & $(0.0 \%)$ & 0.12 \\
\hline Fatigue & 1 & $(10.0 \%)$ & 0 & $(0.0 \%)$ & 1 & $(12.5 \%)$ & 0 & $(0.0 \%)$ & 0.41 \\
\hline Aphtha & 1 & $(10.0 \%)$ & 0 & $(0.0 \%)$ & 2 & $(25.0 \%)$ & 0 & $(0.0 \%)$ & 0.09 \\
\hline \multicolumn{10}{|l|}{ Peripheral Neuropathy } \\
\hline Grade $\geq 1$ & 5 & $(50.0 \%)$ & & & 4 & $(50.0 \%)$ & & & 0.34 \\
\hline Grade $\geq 2$ & 3 & (30.0\%) & & & 2 & $(25.0 \%)$ & & & 0.41 \\
\hline Grade $\geq 3$ & 0 & $(0.0 \%)$ & & & 0 & $(0.0 \%)$ & & & \\
\hline
\end{tabular}

Grade of adverse events were defined according to the CTCAE v3.0 guideline. *P values were calculated with the use of Fisher's exact probability test. Values in parentheses are percentages of row totals.

Table 3. Incidence of peripheral neuropathy during the treatment cycles.

\begin{tabular}{ccccccccccccccccccccccccccccc}
\hline cycles & 1 & 2 & 3 & 4 & 5 & 6 & 7 & 8 & 9 & 10 & 11 & 12 & 13 & 14 & 15 & 16 & 17 & 18 & 19 & 20 & 21 & 22 & 23 & 24 & 25 & 26 & 27 \\
\hline All & & & & & & & & & & & & & & & & & & & & & & & & & & & & \\
Grade 1 & 4 & 7 & 9 & 7 & 5 & 5 & 7 & 5 & 0 & 2 & 1 & 1 & 0 & 1 & 1 & 0 & 0 & 1 & 0 & 0 & 0 & 0 & 0 & 0 & 0 & 0 & 0 \\
Grade 2 & 0 & 1 & 1 & 4 & 5 & 5 & 5 & 5 & 2 & 0 & 1 & 1 & 1 & 1 & 0 & 0 & 1 & 1 & 0 & 0 & 0 & 0 & 1 & 0 & 1 & 1 & 1 \\
Grade 3 & 0 & 0 & 0 & 0 & 0 & 0 & 0 & 0 & 0 & 0 & 0 & 0 & 0 & 0 & 0 & 0 & 0 & 0 & 0 & 0 & 0 & 0 & 0 & 0 & 0 & 0 & 0 \\
$\mathrm{n}$ & 18 & 17 & 17 & 17 & 17 & 17 & 14 & 14 & 9 & 6 & 6 & 4 & 4 & 4 & 4 & 4 & 4 & 2 & 2 & 1 & 1 & 1 & 1 & 1 & 1 & 1 & 1 \\
$\geq 70$ & & & & & & & & & & & & & & & & & & & & & & & & & & & \\
Grade 1 & 2 & 2 & 3 & 4 & 2 & 2 & 3 & 3 & 0 & 1 & 1 & 1 & 0 & 1 & 1 & 0 & 0 & 0 & 0 & 0 & 0 & 0 & 0 & 0 & 0 & 0 & 0 \\
Grade 2 & 0 & 1 & 1 & 1 & 2 & 2 & 1 & 1 & 0 & 0 & 0 & 0 & 0 & 0 & 0 & 0 & 0 & 0 & 0 & 0 & 0 & 0 & 0 & 0 & 0 & 0 & 0 \\
Grade 3 & 0 & 0 & 0 & 0 & 0 & 0 & 0 & 0 & 0 & 0 & 0 & 0 & 0 & 0 & 0 & 0 & 0 & 0 & 0 & 0 & 0 & 0 & 0 & 0 & 0 & 0 & 0 \\
$\mathrm{n}$ & 8 & 7 & 7 & 7 & 7 & 7 & 6 & 6 & 4 & 3 & 3 & 2 & 2 & 2 & 2 & 2 & 2 & 0 & 0 & 0 & 0 & 0 & 0 & 0 & 0 & 0 & 0 \\
\hline
\end{tabular}

Grade of adverse events were defined according to the CTCAE v3.0 guideline.

Two patients in the non-elderly group and one in the elderly group required mFOLFOX6 reintroduction. The reintroduction rate in the eligible patients was $20 \%$ (2 of 6 patients) in the non-elderly group and 33.3\% (1 of 3 patients) in the elderly group. The mFOLFOX6 treatment after maintenance therapy was not reintroduced because of progressive disease on mFOLFOX6 therapy in 2 patients and patient's choice in 4 patients.

\subsection{Response}

An evaluation of the response to treatment was available in 12 patients ( 6 patients in each group). There was no complete response. Tumor response rate was $66.7 \%$ in the non-elderly group and $33.3 \%$ in the elderly group, while the disease control rate was $83.3 \%$ in each group. There was no significant difference in the disease control rate between the two study groups $(\mathrm{P}=1.00)$.

\section{Discussion}

FOLFOX6, in which oxaliplatin at the dose of 100 $\mathrm{mg} / \mathrm{m}^{2}$ was added to the simplified bimonthly regimen with high dose LV followed by bolus and 46 hours continuous infusion of 5-FU every 2 weeks, was originally developed to reduce adverse reactions in 1999 [11]. This regimen is the most useful one among the FOLFOX regimens because it is simple and can be administered on an ambulatory basis. A dosage of $85 \mathrm{mg} / \mathrm{m}^{2}$ of oxaliplatin in the FOLFOX regimen is approved in Japan [12]. Peripheral sensory neuropathy is the cumulative dose-limiting toxicity of oxaliplatin and it usually resolves within a few months after discontinuation of treatment. However, some patients are distressed by this boring reaction for a long time. The reduction of oxaliplatin-induced neuropathy is therefore the current issue in continuing oxaliplatin treatments. Various trials have been conducted and some possible substances, such as $\mathrm{Ca}$ and $\mathrm{Mg}$, carbamazepine, glutathione, alpha lipoic acid, or amifostin have been reported to be useful for the prevention of acute and chronic oxaliplatin-induced neurotoxicity [13-17]. In addition, modification of the oxaliplatin administration schedule may be effective [4]. The notable 
feature of the stop-and-go regimen is the use of oral tegafur-uracil (UFT)/leucovorin (LV) as a maintenance therapy and these oral agents are an effective, well-tolerated, and convenient alternative to i.v. 5-FU [6].

It has been reported that all patients who received mFOLFOX6 treatment developed grade 1 or worse neuropathy during the $9^{\text {th }}$ cycle of treatment and the incidence of grade 2 or worse neuropathy was 30\% during the $10^{\text {th }}$ cycle [3]. The development of neuropathy is dependent on the total dose of oxaliplatin. The incidence of severe neuropathy is about $15 \%$ when the total dose of oxaliplatin reaches $750-800 \mathrm{mg} / \mathrm{m}^{2}$ [18]. The present study used eight cycles of mFOLFOX6 as induction therapy. As a result, only five patients $(27.8 \%)$ had a grade 2 neuropathy and no patient showed grade 3 neuropathy during the $8^{\text {th }}$ cycle of mFOLFOX6 treatment.

This study demonstrated that maintenance therapy with oral daily UFT/LV after mFOLFOX6 is an effective treatment strategy for patients with unresectable advanced colorectal cancer. The median number of cycles of maintenance therapy was 11 cycles. The duration was relatively long and no significant difference in the duration of maintenance therapy was found between the non-elderly and elderly groups, indicating that the maintenance therapy with UFT/LV was not only effective, but also well-tolerated even in elderly patients.

The TTF of mFOLFOX6 therapy for unresectable advanced/recurrent colorectal cancer was 6.4 months and the disease control rate was $83.3 \%$ in this study. In our previous study, which evaluated the efficacy of mFOLFOX6 for elderly patients, the median TTF was 6.2 months in the non-elderly group and 4.9 months in the elderly group and the disease control rate was $100 \%$ and $83.3 \%$ in the non-elderly and elderly patients groups, respectively. [3] Therefore, the efficacy, TTF, and disease control rate of our stop-and-go strategy with discontinuation of oxaliplatin after 8 cycles of the treatment followed by oral UFT/LV were same as continuing oxaliplatin treatment until progression. In addition, discontinuation of oxaliplatin after the $8^{\text {th }}$ cycle reduced the risk of severe toxicity.

Reintroduction of oxaliplatin was scheduled in the OPTIMOX1 study, and the oxaliplatin reintroduction rate was as low as $40.1 \%$ [4]. The main reason for the low reintroduction rate was the patient's refusal to resume the oxaliplatin-including regimen because of its severe adverse events including peripheral neuropathy. Therefore, maintenance therapy with daily oral UFT/LV was continued until disease progression or toxicity in this study. Nevertheless, oxaliplatin reintroduction was unsuccessful, namely, the reintroduction rate was $20 \%$ in the non-elderly group and $33.3 \%$ in the elderly group. The major reason was the patient choice (66.7\%). Just after the start of the present study, bevacizumab was approved for colorectal cancer patients in Japan. Therefore, most patients desired another regimen using bevacizumab when disease progression was recognized.

The average life span has been prolonged in the world and elderly colorectal patients are increasing. Generally, the pharmacokinetics of drugs deteriorates in the elderly because of organ dysfunction associated with aging [19, 20]. Some articles reported that severe neutropenia and thrombocytopenia tend to occur in elderly patients [21]. Meanwhile, the complete administration of the drugs as planned, including 5-FU/LV, irinotecan, and oxaliplatin, prolongs survival in patients with colon cancer [22]. Therefore, it is important to establish a safe regimen using active cytotoxic agents for elderly patients. The present study investigated the safety of this stop-and-go regimen in elderly patients over 70 years of age. The incidence of grade 3 or more neutropenia in elderly patients was similar to that seen in non-elderly patients ( $37.5 \%$ vs. $40.0 \%, \mathrm{P}=0.380$ ). In addition, the incidence of grade 2 peripheral neuropathy in elderly patients (25.0\%) was equivalent to that in non-elderly patients (30.0\%). These findings indicated that this stop-and-go strategy is feasible for elderly patients with unresectable/recurrent colorectal cancer. In conclusion, the results of the present study demonstrated that 8 cycles of $\mathrm{m}$ FOLFOX6 is the optimal duration for the treatment of patients with advanced colorectal cancer and stop-and-go strategy is tolerable and effective even in elderly patients. Further study is warranted to establish novel stop-and-go regimens, including those using molecular targeting drugs.

\section{Conclusion}

The results of this study demonstrated that mFOLFOX6 stop-and-go regimen with oral UFT/LV maintenance therapy is well-tolerated and effective even in elderly patients with unresectable advanced colorectal cancer.

\section{Acknowledgment}

We deeply appreciate Dr. Kouji Kodama (Department of Radiology, Shimane Prefectural Central Hospital) for his valuable assistance and extramural review.

\section{Disclosure Statement}

The authors declare that they have no conflict of interests.

\section{Conflict-of-Interest Notification Field}

The authors declare that they have no conflict of interests. 


\section{REFERENCES}

[1] I. Hyodo, et al., "Present Status and Perspectives of Colorectal Cancer in Asia: Colorectal Cancer Working Group report in 30th Asia-Pacific Cancer Conference,” Japanese Journal of Clinical Oncology, Vol. 40, Suppl. 1, 2010, pp. I38-I43. http://dx.doi.org/10.1093/jico/hyq125

[2] R. M. Goldberg, et al., “A Randomized Controlled Trial of Fluorouracil Plus Leucovorin, Irinotecan, and Oxaliplatin Combinations in Patients with Previously Untreated Metastatic Colorectal Cancer," Journal of Clinical Oncology, Vol. 22, No. 1, 2004, pp. 23-30.

http://dx.doi.org/10.1200/JCO.2004.09.046

[3] S. Sugimoto, et al., "Multicenter Safety Study of mFOLFOX6 for Unresectable Advanced/Recurrent Colorectal Cancer in Elderly Patients,” Journal of Experimental \& Clinical Cancer Research, Vol. 28, 2009, p. 109. http://dx.doi.org/10.1186/1756-9966-28-109

[4] C. Tournigand, et al., "OPTIMOX1: A Randomized Study of FOLFOX4 or FOLFOX7 with Oxaliplatin in a Stop-and-Go Fashion in Advanced Colorectal Cancer-A GERCOR Study,” Journal of Clinical Oncology, Vol. 24, No. 3, 2006, pp. 394-400. http://dx.doi.org/10.1200/JCO.2005.03.0106

[5] J. F. Bosset, et al., "Preoperative Radiochemotherapy in Rectal Cancer: Long-Term Results of a Phase II Trial," International Journal of Radiation Oncology *Biology* Physics, Vol. 46, No. 2, 2000, pp. 323-327. http://dx.doi.org/10.1016/S0360-3016(99)00411-3

[6] M. M. Borner, et al., "Patient Preference and Pharmacokinetics of Oral Modulated UFT versus Intravenous Fluorouracil and Leucovorin: A Randomised Crossover Trial in Advanced Colorectal Cancer," European Journal of Cancer, Vol. 38, No. 3, 2002, pp. 349-358. http://dx.doi.org/10.1016/S0959-8049(01)00371-9

[7] M. o. H. LaW, "Ethical Guidelines for Clinical Studies," http://www.med.or.jp/wma/helsinki08_j.html

[8] E. P. f. M. R. I. H. S., "18th WMA General Assembly. World Medical Association Declaration of Helsinki," 2008. http://www.med.or.jp/wma/helsinki08_j.html

[9] I. NC, Common Terminology Criteria for Adverse Events v3.0, 2006.

[10] P. Therasse, et al., "New Guidelines to Evaluate the Response to Treatment in Solid Tumors. European Organization for Research and Treatment of Cancer, National Cancer Institute of the United States, National Cancer Institute of Canada," Journal of the National Cancer Institute, Vol. 92, No. 3, 2000, pp. 205-216.

http://dx.doi.org/10.1093/jnci/92.3.205

[11] F. Maindrault-Goebel, et al., "Oxaliplatin Added to the Simplified Bimonthly Leucovorin and 5-Fluorouracil Regimen as Second-Line Therapy for Metastatic Colorectal Cancer (FOLFOX6). GERCOR,” European Journal of Cancer, Vol. 35, No. 9, 1999, pp. 1338-1342. http://dx.doi.org/10.1016/S0959-8049(99)00149-5
[12] T. Shimizu, et al., "Oxaliplatin/Fluorouracil/Leucovorin (FOLFOX4 and Modified FOLFOX6) in Patients with Refractory or Advanced Colorectal Cancer: Post-Approval Japanese Population Experience,” International Journal of Clinical Oncology, Vol. 12, No. 3, 2007, pp. 218223. http://dx.doi.org/10.1007/s10147-007-0658-x

[13] L. Gamelin, et al., "Prevention of Oxaliplatin-Related Neurotoxicity by Calcium and Magnesium Infusions: A Retrospective Study of 161 Patients Receiving Oxaliplatin Combined with 5-Fluorouracil and Leucovorin for Advanced Colorectal Cancer," Clinical Cancer Research, Vol. 10, No. 12 Pt 1, 2004, pp. 4055-4061.

[14] F. Eckel, et al., "Prevention of Oxaliplatin-Induced Neuropathy by Carbamazepine. A Pilot Study,” Dtsch Med Wochenschr, Vol. 127, No. 3, 2002, pp. 78-82. http://dx.doi.org/10.1055/s-2002-19594

[15] S. Cascinu, et al., "Neuroprotective Effect of Reduced Glutathione on Oxaliplatin-Based Chemotherapy in Advanced Colorectal Cancer: A Randomized, Double-Blind, Placebo-Controlled Trial,” Journal of Clinical Oncology, Vol. 20, No. 16, 2002, pp. 3478-3483. http://dx.doi.org/10.1200/JCO.2002.07.061

[16] M. Penz, et al., "Subcutaneous Administration of Amifostine: A Promising Therapeutic Option in Patients with Oxaliplatin-Related Peripheral Sensitive Neuropathy,” Annals of Oncology, Vol. 12, No. 3, 2001, pp. 421-422. http://dx.doi.org/10.1023/A:1011184609963

[17] C. Gedlicka, et al., "Effective Treatment of Oxaliplatin-Induced Cumulative Polyneuropathy with Alpha-Lipoic Acid,” Journal of Clinical Oncology, Vol. 20, No. 15, 2002, pp. 3359-3361. http://dx.doi.org/10.1200/JCO.2002.99.502

[18] A. Grothey, "Oxaliplatin-Safety Profile: Neurotoxicity," Semin Oncol, Vol. 30, No. 4, Suppl. 15, 2003, pp. 5-13. http://dx.doi.org/10.1016/S0093-7754(03)00399-3

[19] S. C. Montamat, B. J. Cusack and R. E. Vestal, "Management of Drug Therapy in the Elderly," The New England Journal of Medicine, Vol. 321, No. 5, 1989, pp. 303309. http://dx.doi.org/10.1056/NEJM198908033210507

[20] D. J. Greenblatt, E. M. Sellers and R. I. Shader, "Drug Therapy: Drug Disposition in Old Age," The New England Journal of Medicine, Vol. 306, No. 18, 1982, pp. 1081-1088. http://dx.doi.org/10.1056/NEJM198205063061804

[21] R. M. Goldberg, et al., "Pooled Analysis of Safety and Efficacy of Oxaliplatin plus Fluorouracil/Leucovorin Administered Bimonthly in Elderly Patients with Colorectal Cancer," Journal of Clinical Oncology, Vol. 24, No. 25, 2006, pp. 4085-4091.

http://dx.doi.org/10.1200/JCO.2006.06.9039

[22] A. Grothey, et al., "Survival of Patients with Advanced Colorectal Cancer Improves with the Availability of Fluorouracil-Leucovorin, Irin Otecan, and Oxaliplatin in the Course of Treatment," Journal of Clinical Oncology, Vol. 22, No. 7, 2004, pp. 1209-1214. http://dx.doi.org/10.1200/JCO.2004.11.037 


\section{List of Abbreviations}

FOLFOX: oxaliplatin plus -fluorouracil/leucovorin UFT: uracil-tegafur

LV: leucovorin

TTF: treatment failure 